\title{
On oscillation of second-order neutral dynamic equations
}

\author{
Tao Ji ${ }^{1}$, Shuhong Tang ${ }^{1 *}$ and Ethiraju Thandapani ${ }^{2}$
}

\section{${ }^{*}$ Correspondence:}

wfxytang@163.com

1 School of Information and Control Engineering, Weifang University, Weifang, Shandong 261061, P.R. China

Full list of author information is available at the end of the article

\begin{abstract}
Assuming

$$
\int_{t_{0}}^{\infty} \frac{\Delta t}{r^{1 / \gamma}(t)}<\infty
$$

a new oscillation criterion for a half-linear second-order neutral dynamic equation

$$
\left(r(t)\left((x(t)+p(t) x(t-\tau))^{\Delta}\right)^{\gamma}\right)^{\Delta}+q(t)|x(t-\delta)|^{\gamma-1} x(t-\delta)=0
$$

is presented. An interesting example is provided to show that the delayed function $\delta(t)=t-\delta$ plays an important role in the oscillatory behavior.
\end{abstract}

MSC: 34K11;34N05; 39A10

Keywords: oscillation; neutral dynamic equation; time scale

\section{Introduction}

This work is concerned with the oscillatory behavior of solutions to a second-order halflinear neutral delay dynamic equation

$$
\left(r(t)\left((x(t)+p(t) x(t-\tau))^{\Delta}\right)^{\gamma}\right)^{\Delta}+q(t)|x(t-\delta)|^{\gamma-1} x(t-\delta)=0
$$

on a time scale $\mathbb{T}$, where $t \in\left[t_{0}, \infty\right)_{\mathbb{T}}:=\left[t_{0}, \infty\right) \cap \mathbb{T}$. In what follows, we assume that $\gamma \geq 1$ is a quotient of odd positive integers, $\tau \geq 0, \delta \geq 0, t-\tau: \mathbb{T} \rightarrow \mathbb{T}, t-\delta: \mathbb{T} \rightarrow \mathbb{T}, r, p$, and $q$ are real-valued rd-continuous functions defined on $\mathbb{T}$ such that $r(t)>0, \int_{t_{0}}^{\infty} r^{-1 / \gamma}(t) \Delta t<\infty$, $0 \leq p(t) \leq p_{0}<\infty$, and $q(t)>0$ for $t \in\left[t_{0}, \infty\right)_{\mathbb{T}}$.

By a solution of (1.1) we mean a nontrivial real-valued function $x$ which has the properties $x(t)+p(t) x(t-\tau) \in \mathrm{C}_{\mathrm{rd}}^{1}\left[t_{x}, \infty\right)_{\mathbb{T}}$ and $r(t)\left((x(t)+p(t) x(t-\tau))^{\Delta}\right)^{\gamma} \in \mathrm{C}_{\mathrm{rd}}^{1}\left[t_{x}, \infty\right)_{\mathbb{T}}, t_{x} \in$ $\left[t_{0}, \infty\right)_{\mathbb{T}}$ and satisfying (1.1) for all $t \in\left[t_{x}, \infty\right)_{\mathbb{T}}$. Our attention is restricted to those solutions of $(1.1)$ which exist on some half-line $\left[t_{x}, \infty\right)_{\mathbb{T}}$ and satisfy $\sup \left\{|x(t)|: t \in\left[t_{1}, \infty\right)_{\mathbb{T}}\right\}>0$ for any $t_{1} \in\left[t_{x}, \infty\right)_{\mathbb{T}}$. A solution $x$ of $(1.1)$ is said to be oscillatory if it is neither eventually positive nor eventually negative; otherwise, it is called nonoscillatory. Equation (1.1) is said to be oscillatory if all its solutions are oscillatory.

The theory of time scales, which has recently received a lot of attention, was introduced by Hilger in order to unify continuous and discrete analysis (see Hilger [1]). Several authors have expounded on various aspects of this new theory; see the survey paper by Agarwal 
et al. [2] and the references cited therein. The books on the subject of time scales, by Bohner and Peterson [3, 4], summarize and organize much of time scale calculus and some applications of dynamic equations on time scales.

In recent years, there has been much research activity concerning the oscillatory and nonoscillatory behavior of solutions to various classes of differential, difference, and dynamic equations. We refer the reader to [5-28] and the references therein. If $\mathbb{T}=\mathbb{R}$, then $\sigma(t)=t, \mu(t)=0, x^{\Delta}(t)=x^{\prime}(t), \int_{a}^{b} f(t) \Delta t=\int_{a}^{b} f(t) \mathrm{d} t$, and equation (1.1) becomes a secondorder neutral delay differential equation

$$
\left(r(t)\left((x(t)+p(t) x(t-\tau))^{\prime}\right)^{\gamma}\right)^{\prime}+q(t)|x(t-\delta)|^{\gamma-1} x(t-\delta)=0 .
$$

If $\mathbb{T}=\mathbb{Z}$, then $\sigma(t)=t+1, \mu(t)=1, x^{\Delta}(t)=x(t+1)-x(t), \int_{a}^{b} f(t) \Delta t=\sum_{t=a}^{b-1} f(t)$, and equation (1.1) reduces to a second-order neutral delay difference equation

$$
\Delta\left(r(t)(\Delta(x(t)+p(t) x(t-\tau)))^{\gamma}\right)+q(t)|x(t-\delta)|^{\gamma-1} x(t-\delta)=0 .
$$

If $\mathbb{T}=h \mathbb{Z}$ where $h>0$, then $\sigma(t)=t+h, \mu(t)=h, x^{\Delta}(t)=\Delta_{h} x(t)=(x(t+h)-x(t)) / h$, $\int_{a}^{b} f(t) \Delta t=\sum_{k=0}^{(b-a-h) / h} f(a+k h) h$, and equation (1.1) becomes a second-order neutral delay difference equation

$$
\Delta_{h}\left(r(t)\left(\Delta_{h}(x(t)+p(t) x(t-\tau))\right)^{\gamma}\right)+q(t)|x(t-\delta)|^{\gamma-1} x(t-\delta)=0 .
$$

In what follows, we present some background details that motivate the contents of this note. Jiang and $\mathrm{Li} \mathrm{[5]} \mathrm{studied} \mathrm{oscillatory} \mathrm{properties} \mathrm{of} \mathrm{equation} \mathrm{(1.2)} \mathrm{in} \mathrm{the} \mathrm{case}$ $\int_{t_{0}}^{\infty} r^{-1 / \gamma}(t) \mathrm{d} t=\infty$. Saker [6] and Sun and Saker [7] considered oscillation of equation (1.3) and established some criteria under the assumption that $\sum_{t=t_{0}}^{\infty} r^{-1 / \gamma}(t)=\infty$. To the best of our knowledge, there are few results for oscillation of equation (1.3) in the case where $\sum_{i=t_{0} / h}^{\infty} r^{-1 / \gamma}(i h)<\infty$. Agarwal et al. [9], Saker [12], and Tripathy [15] studied oscillatory behavior of (1.1) in the case where $\int_{t_{0}}^{\infty} r^{-1 / \gamma}(t) \Delta t=\infty$. In particular, Tripathy [15] established some oscillation tests for (1.1) assuming that $0 \leq p(t) \leq p_{0}<\infty$.

The purpose of this paper is to obtain a new sufficient condition for oscillation of (1.1). The result obtained is essentially new for equations (1.2)-(1.4). This paper is organized as follows. In Section 2, we use the Riccati transformation technique to prove the main results. In Section 3, we apply our criterion in equations (1.2)-(1.4) to establish some new oscillation criteria.

\section{Oscillation results}

To prove the main results, we use the formula

$$
\left(y^{\gamma}\right)^{\Delta}(t)=\gamma \int_{0}^{1}\left[h y^{\sigma}(t)+(1-h) y(t)\right]^{\gamma-1} y^{\Delta}(t) \mathrm{d} h,
$$

which is a simple consequence of Keller's chain rule (see [3, Theorem 1.90]). We also need the following lemma.

Lemma 2.1 (See [22, Lemma 1]) Assume $\gamma \geq 1$ and $a, b \in \mathbb{R}$. If $a \geq 0, b \geq 0$, then

$$
a^{\gamma}+b^{\gamma} \geq \frac{(a+b)^{\gamma}}{2^{\gamma-1}}
$$


In what follows, all functional inequalities are assumed to be satisfied for all sufficiently large $t$.

Theorem 2.2 Let $\tilde{\mathbb{T}}:=\{t-\tau: t \in \mathbb{T}\}$. Suppose also that

$$
\int_{t_{0}}^{\infty} Q(t) \Delta t=\infty
$$

and

$$
\limsup _{t \rightarrow \infty} \int_{t_{0}}^{t}\left[\frac{f^{\gamma}(\sigma(s)) Q(s)}{2^{\gamma-1}}-\left(1+p_{0}^{\gamma}\right)\left(\frac{\gamma}{\gamma+1}\right)^{\gamma+1} \frac{(f(s))^{\gamma^{2}-1}}{f^{\gamma^{2}}(\sigma(s)) r^{1 / \gamma}(s)}\right] \Delta s=\infty,
$$

where

$$
Q(t):=\min \{q(t), q(t-\tau)\} \text { and } f(t):=\int_{t}^{\infty} \frac{\Delta s}{r^{1 / \gamma}(s)}
$$

Then equation (1.1) is oscillatory.

Proof Suppose to the contrary that (1.1) has a nonoscillatory solution $x$. Without loss of generality, we may assume that there exists $t_{1} \in\left[t_{0}, \infty\right)_{\mathbb{T}}$ such that $x(t-N)>0$ for all $t \in\left[t_{1}, \infty\right)_{\mathbb{T}}$, where $N:=\max \{\tau, \delta\}$. Set

$$
z(t):=x(t)+p(t) x(t-\tau) .
$$

Then $z(t)>0$ and

$$
\left(r(t)\left(z^{\Delta}(t)\right)^{\gamma}\right)^{\Delta}=-q(t) x^{\gamma}(t-\delta)<0
$$

for $t \in\left[t_{1}, \infty\right)_{\mathbb{T}}$. Hence, $r\left(z^{\Delta}\right)^{\gamma}$ is of one sign. On the other hand, we have by (1.1) and [3, Theorem 1.93] that

$$
\begin{aligned}
& \left(r(t)\left(z^{\Delta}(t)\right)^{\gamma}\right)^{\Delta}+q(t) x^{\gamma}(t-\delta)+p_{0}^{\gamma} q(t-\tau) x^{\gamma}(t-\tau-\delta) \\
& \quad+p_{0}^{\gamma}\left(r(t-\tau)\left(z^{\Delta}(t-\tau)\right)^{\gamma}\right)^{\Delta}=0 .
\end{aligned}
$$

Thus, we get by (2.2) that

$$
\left(r(t)\left(z^{\Delta}(t)\right)^{\gamma}\right)^{\Delta}+p_{0}^{\gamma}\left(r(t-\tau)\left(z^{\Delta}(t-\tau)\right)^{\gamma}\right)^{\Delta}+\frac{Q(t)}{2^{\gamma-1}} z^{\gamma}(t-\delta) \leq 0 .
$$

If $z^{\Delta}>0$, then by (2.5) there exist a constant $k>0$ and $t_{2} \in\left[t_{1}, \infty\right)_{\mathbb{T}}$ such that for $t \in$ $\left[t_{2}, \infty\right)_{\mathbb{T}}$,

$$
Q(t) \leq-k\left(r(t)\left(z^{\Delta}(t)\right)^{\gamma}\right)^{\Delta}-k p_{0}^{\gamma}\left(r(t-\tau)\left(z^{\Delta}(t-\tau)\right)^{\gamma}\right)^{\Delta} .
$$


Integrating the latter inequality from $t_{2}$ to $t$, we have

$$
\begin{aligned}
\int_{t_{2}}^{t} Q(s) \Delta s \leq & k\left(r\left(t_{2}\right)\left(z^{\Delta}\left(t_{2}\right)\right)^{\gamma}-r(t)\left(z^{\Delta}(t)\right)^{\gamma}\right) \\
& +k p_{0}^{\gamma}\left(r\left(t_{2}-\tau\right)\left(z^{\Delta}\left(t_{2}-\tau\right)\right)^{\gamma}-r(t-\tau)\left(z^{\Delta}(t-\tau)\right)^{\gamma}\right) \\
\leq & k r\left(t_{2}\right)\left(z^{\Delta}\left(t_{2}\right)\right)^{\gamma}+k p_{0}^{\gamma} r\left(t_{2}-\tau\right)\left(z^{\Delta}\left(t_{2}-\tau\right)\right)^{\gamma}
\end{aligned}
$$

which contradicts (2.3).

Assume now that $z^{\Delta}<0$. Define a Riccati substitution

$$
\omega(t):=\frac{r(t)\left(z^{\Delta}(t)\right)^{\gamma}}{z^{\gamma}(t)}
$$

Then $\omega(t)<0$. Noticing that $r\left(z^{\Delta}\right)^{\gamma}$ is decreasing, we have

$$
(r(s))^{1 / \gamma} z^{\Delta}(s) \leq(r(t))^{1 / \gamma} z^{\Delta}(t), \quad s \geq t
$$

Dividing the latter inequality by $(r(s))^{1 / \gamma}$ and integrating the resulting inequality from $t$ to $l$, we get

$$
z(l) \leq z(t)+(r(t))^{1 / \gamma} z^{\Delta}(t) \int_{t}^{l}\left(\frac{1}{r(s)}\right)^{1 / \gamma} \Delta s .
$$

Note that $\lim _{l \rightarrow \infty} z(l) \geq 0$. Letting $l \rightarrow \infty$ in the above inequality, we obtain $0 \leq z(t)+$ $(r(t))^{1 / \gamma} z^{\Delta}(t) f(t)$, which implies that

$$
\frac{(r(t))^{1 / \gamma} z^{\Delta}(t)}{z(t)} f(t) \geq-1
$$

Then we have by (2.6) that

$$
-1 \leq \omega(t) f^{\gamma}(t) \leq 0 .
$$

Similarly, we define another Riccati transformation

$$
u(t):=\frac{r(t-\tau)\left(z^{\Delta}(t-\tau)\right)^{\gamma}}{z^{\gamma}(t)}
$$

Then $u(t)<0$. Noting that $r\left(z^{\Delta}\right)^{\gamma}$ is decreasing, we have $r(t-\tau)\left(z^{\Delta}(t-\tau)\right)^{\gamma} \geq r(t)\left(z^{\Delta}(t)\right)^{\gamma}$, and so $u(t) \geq \omega(t)$. From (2.7), we have

$$
-1 \leq u(t) f^{\gamma}(t) \leq 0
$$

Differentiating (2.6), we get

$$
\omega^{\Delta}(t)=\frac{\left(r(t)\left(z^{\Delta}(t)\right)^{\gamma}\right)^{\Delta} z^{\gamma}(t)-r(t)\left(z^{\Delta}(t)\right)^{\gamma}\left(z^{\gamma}(t)\right)^{\Delta}}{z^{\gamma}(t) z^{\gamma}(\sigma(t))} .
$$


By virtue of (2.1), we obtain

$$
\left(z^{\gamma}(t)\right)^{\Delta} \leq \gamma z^{\Delta}(t)\left(z^{\sigma}(t)\right)^{\gamma-1}
$$

Using (2.11) in (2.10), we have

$$
\omega^{\Delta}(t) \leq \frac{\left(r(t)\left(z^{\Delta}(t)\right)^{\gamma}\right)^{\Delta}}{z^{\gamma}(\sigma(t))}-\gamma \frac{r(t)\left(z^{\Delta}(t)\right)^{\gamma+1}}{z^{\gamma}(t) z(\sigma(t))} .
$$

By (2.6) and the latter inequality, we find

$$
\omega^{\Delta}(t) \leq \frac{\left(r(t)\left(z^{\Delta}(t)\right)^{\gamma}\right)^{\Delta}}{z^{\gamma}(\sigma(t))}-\gamma \frac{(\omega(t))^{(\gamma+1) / \gamma}}{(r(t))^{1 / \gamma}}
$$

due to $z(t) \geq z(\sigma(t))$. Differentiating (2.8), we obtain

$$
u^{\Delta}(t)=\frac{\left(r(t-\tau)\left(z^{\Delta}(t-\tau)\right)^{\gamma}\right)^{\Delta} z^{\gamma}(t)-r(t-\tau)\left(z^{\Delta}(t-\tau)\right)^{\gamma}\left(z^{\gamma}(t)\right)^{\Delta}}{z^{\gamma}(t) z^{\gamma}(\sigma(t))} .
$$

Using (2.11) in (2.13), we have

$$
\begin{aligned}
u^{\Delta}(t) \leq & \frac{\left(r(t-\tau)\left(z^{\Delta}(t-\tau)\right)^{\gamma}\right)^{\Delta}}{z^{\gamma}(\sigma(t))} \\
& -\gamma \frac{r(t-\tau)\left(z^{\Delta}(t-\tau)\right)^{\gamma} z^{\Delta}(t)}{z^{\gamma}(t) z(\sigma(t))} .
\end{aligned}
$$

From (2.8), (2.14), and the fact that $r(t)\left(z^{\Delta}(t)\right)^{\gamma} \leq r(t-\tau)\left(z^{\Delta}(t-\tau)\right)^{\gamma}$, we get

$$
u^{\Delta}(t) \leq \frac{\left(r(t-\tau)\left(z^{\Delta}(t-\tau)\right)^{\gamma}\right)^{\Delta}}{z^{\gamma}(\sigma(t))}-\gamma \frac{(u(t))^{(\gamma+1) / \gamma}}{(r(t))^{1 / \gamma}} .
$$

Thus, by (2.12) and (2.15), we have

$$
\begin{aligned}
\omega^{\Delta}(t)+p_{0}^{\gamma} u^{\Delta}(t) \leq & \frac{\left(r(t)\left(z^{\Delta}(t)\right)^{\gamma}\right)^{\Delta}+p_{0}^{\gamma}\left(r(t-\tau)\left(z^{\Delta}(t-\tau)\right)^{\gamma}\right)^{\Delta}}{z^{\gamma}(\sigma(t))} \\
& -\gamma \frac{(\omega(t))^{(\gamma+1) / \gamma}}{(r(t))^{1 / \gamma}}-p_{0}^{\gamma} \gamma \frac{(u(t))^{(\gamma+1) / \gamma}}{(r(t))^{1 / \gamma}} .
\end{aligned}
$$

Using $z(t-\delta) \geq z(\sigma(t))$ and (2.5) yields

$$
\omega^{\Delta}(t)+p_{0}^{\gamma} u^{\Delta}(t) \leq-\frac{Q(t)}{2^{\gamma-1}}-\gamma \frac{(\omega(t))^{(\gamma+1) / \gamma}}{(r(t))^{1 / \gamma}}-p_{0}^{\gamma} \gamma \frac{(u(t))^{(\gamma+1) / \gamma}}{(r(t))^{1 / \gamma}} .
$$

Multiplying the latter inequality by $f^{\gamma}(\sigma(t))$ and integrating the resulting inequality on $\left[t_{2}, t\right]_{\mathbb{T}}\left(t_{2} \in\left[t_{1}, \infty\right)_{\mathbb{T}}\right.$ sufficiently large), we obtain

$$
\begin{gathered}
\omega(t) f^{\gamma}(t)-\omega\left(t_{2}\right) f^{\gamma}\left(t_{2}\right)-\int_{t_{2}}^{t}\left(f^{\gamma}(s)\right)^{\Delta} \omega(s) \Delta s+p_{0}^{\gamma} u(t) f^{\gamma}(t) \\
-p_{0}^{\gamma} u\left(t_{2}\right) f^{\gamma}\left(t_{2}\right)-p_{0}^{\gamma} \int_{t_{2}}^{t}\left(f^{\gamma}(s)\right)^{\Delta} u(s) \Delta s
\end{gathered}
$$


di et al. Advances in Difference Equations 2013, 2013:340

Page 6 of 9

http://www.advancesindifferenceequations.com/content/2013/1/340

$$
\begin{aligned}
\leq & -\int_{t_{2}}^{t} \frac{f^{\gamma}(\sigma(s)) Q(s)}{2^{\gamma-1}} \Delta s-\gamma \int_{t_{2}}^{t} \frac{(\omega(s))^{(\gamma+1) / \gamma}}{(r(s))^{1 / \gamma}} f^{\gamma}(\sigma(s)) \Delta s \\
& -\gamma p_{0}^{\gamma} \int_{t_{2}}^{t} \frac{(u(s))^{(\gamma+1) / \gamma}}{(r(s))^{1 / \gamma}} f^{\gamma}(\sigma(s)) \Delta s .
\end{aligned}
$$

Hence, by (2.1) and $f^{\Delta}=-r^{-1 / \gamma}<0$, we get

$$
\int_{t_{2}}^{t}\left(f^{\gamma}(s)\right)^{\Delta} \omega(s) \Delta s \leq-\gamma \int_{t_{2}}^{t}(f(s))^{\gamma-1} \frac{\omega(s)}{(r(s))^{1 / \gamma}} \Delta s .
$$

Similarly, we have

$$
\int_{t_{2}}^{t}\left(f^{\gamma}(s)\right)^{\Delta} u(s) \Delta s \leq-\gamma \int_{t_{2}}^{t}(f(s))^{\gamma-1} \frac{u(s)}{(r(s))^{1 / \gamma}} \Delta s .
$$

Therefore, we obtain by (2.16) that

$$
\begin{aligned}
& \gamma \int_{t_{2}}^{t}(f(s))^{\gamma-1} \frac{\omega(s)}{(r(s))^{1 / \gamma}} \Delta s+\gamma \int_{t_{2}}^{t} \frac{(\omega(s))^{(\gamma+1) / \gamma}}{(r(s))^{1 / \gamma}} f^{\gamma}(\sigma(s)) \Delta s \\
& \quad+\gamma p_{0}^{\gamma} \int_{t_{2}}^{t}(f(s))^{\gamma-1} \frac{u(s)}{(r(s))^{1 / \gamma}} \Delta s+\gamma p_{0}^{\gamma} \int_{t_{2}}^{t} \frac{(u(s))^{(\gamma+1) / \gamma}}{(r(s))^{1 / \gamma}} f^{\gamma}(\sigma(s)) \Delta s \\
& \quad+\int_{t_{2}}^{t} \frac{f^{\gamma}(\sigma(s)) Q(s)}{2^{\gamma-1}} \Delta s \\
& \leq-\omega(t) f^{\gamma}(t)+\omega\left(t_{2}\right) f^{\gamma}\left(t_{2}\right)-p_{0}^{\gamma} u(t) f^{\gamma}(t)+p_{0}^{\gamma} u\left(t_{2}\right) f^{\gamma}\left(t_{2}\right) .
\end{aligned}
$$

Let $\chi=-\omega(t)$,

$$
A=\frac{\gamma f^{\gamma}(\sigma(s))}{(r(s))^{1 / \gamma}}, \quad \text { and } \quad B=\frac{\gamma(f(s))^{\gamma-1}}{(r(s))^{1 / \gamma}}
$$

Using the inequality

$$
A \chi^{(\gamma+1) / \gamma}-B \chi \geq-\frac{\gamma^{\gamma}}{(\gamma+1)^{\gamma+1}} \frac{B^{\gamma+1}}{A^{\gamma}}
$$

where $A>0$ is a constant, we get

$$
\begin{gathered}
\gamma \int_{t_{2}}^{t}(f(s))^{\gamma-1} \frac{\omega(s)}{(r(s))^{1 / \gamma}} \Delta s+\gamma \int_{t_{2}}^{t} \frac{(\omega(s))^{(\gamma+1) / \gamma}}{(r(s))^{1 / \gamma}} f^{\gamma}(\sigma(s)) \Delta s \\
\geq-\left(\frac{\gamma}{\gamma+1}\right)^{\gamma+1} \int_{t_{2}}^{t} \frac{(f(s))^{\gamma^{2}-1}}{f \gamma^{2}(\sigma(s)) r^{1 / \gamma}(s)} \Delta s .
\end{gathered}
$$

Similarly, we have

$$
\begin{gathered}
\gamma \int_{t_{2}}^{t}(f(s))^{\gamma-1} \frac{u(s)}{(r(s))^{1 / \gamma}} \Delta s+\gamma \int_{t_{2}}^{t} \frac{(u(s))^{(\gamma+1) / \gamma}}{(r(s))^{1 / \gamma}} f^{\gamma}(\sigma(s)) \Delta s \\
\geq-\left(\frac{\gamma}{\gamma+1}\right)^{\gamma+1} \int_{t_{2}}^{t} \frac{(f(s))^{\gamma^{2}-1}}{f \gamma^{2}(\sigma(s)) r^{1 / \gamma}(s)} \Delta s .
\end{gathered}
$$


Therefore, (2.17) implies that

$$
\begin{aligned}
& \int_{t_{2}}^{t}\left[\frac{f^{\gamma}(\sigma(s)) Q(s)}{2^{\gamma-1}}-\left(1+p_{0}^{\gamma}\right)\left(\frac{\gamma}{\gamma+1}\right)^{\gamma+1} \frac{(f(s))^{\gamma^{2}-1}}{f^{\gamma^{2}}(\sigma(s)) r^{1 / \gamma}(s)}\right] \Delta s \\
& \leq-\omega(t) f^{\gamma}(t)-p_{0}^{\gamma} u(t) f^{\gamma}(t) \leq 1+p_{0}^{\gamma}
\end{aligned}
$$

due to (2.7) and (2.9), which contradicts (2.4). The proof is complete.

Remark 2.3 Theorem 2.2 complements the results obtained in [23] since it can be applied in the case where $\gamma>1$.

\section{Applications}

Due to Theorem 2.2, we present the following results for oscillation of equations (1.2)(1.4).

Corollary 3.1 Assume $\mathbb{T}=\mathbb{R}$ and let

$$
\int_{t_{0}}^{\infty} Q(t) \mathrm{d} t=\infty
$$

and

$$
\limsup _{t \rightarrow \infty} \int_{t_{0}}^{t}\left[\frac{\theta^{\gamma}(s) Q(s)}{2^{\gamma-1}}-\left(1+p_{0}^{\gamma}\right)\left(\frac{\gamma}{\gamma+1}\right)^{\gamma+1} \frac{1}{\theta(s) r^{1 / \gamma}(s)}\right] \mathrm{d} s=\infty,
$$

where $Q$ is defined as in Theorem 2.2 and

$$
\theta(t):=\int_{t}^{\infty} \frac{\mathrm{d} s}{r^{1 / \gamma}(s)}
$$

Then equation (1.2) oscillates.

Corollary 3.2 Assume $\mathbb{T}=\mathbb{Z}$ and let

$$
\sum_{t=t_{0}}^{\infty} Q(t)=\infty
$$

and

$$
\limsup _{t \rightarrow \infty} \sum_{s=t_{0}}^{t-1}\left[\frac{\beta^{\gamma}(s+1) Q(s)}{2^{\gamma-1}}-\left(1+p_{0}^{\gamma}\right)\left(\frac{\gamma}{\gamma+1}\right)^{\gamma+1} \frac{(\beta(s))^{\gamma^{2}-1}}{\beta^{\gamma^{2}}(s+1) r^{1 / \gamma}(s)}\right]=\infty,
$$

where $Q$ is defined as in Theorem 2.2 and

$$
\beta(t):=\sum_{s=t}^{\infty} \frac{1}{r^{1 / \gamma}(s)}
$$

Then equation (1.3) oscillates. 
Corollary 3.3 Assume $\mathbb{T}=h \mathbb{Z}, h>0$, and let

$$
\sum_{i=\frac{t_{0}}{h}}^{\infty} Q(i h)=\infty
$$

and

$$
\limsup _{t \rightarrow \infty} \sum_{i=\frac{t_{0}}{h}}^{\frac{t}{h}-1}\left[\frac{\xi^{\gamma}((i+1) h) Q(i h)}{2^{\gamma-1}}-\left(1+p_{0}^{\gamma}\right)\left(\frac{\gamma}{\gamma+1}\right)^{\gamma+1} \frac{(\xi(i h))^{\gamma^{2}-1}}{\xi \gamma^{2}((i+1) h) r^{1 / \gamma}(i h)}\right]=\infty
$$

where $Q$ is defined as in Theorem 2.2 and

$$
\xi(t):=\sum_{i=\frac{t}{h}}^{\infty} \frac{1}{r^{1 / \gamma}(i h)}
$$

Then equation (1.4) oscillates.

Example 3.4 For $t \geq 1$, consider a second-order neutral delay differential equation

$$
\left(\mathrm{e}^{2 t}\left(x(t)+\frac{1}{2} x(t-2)\right)^{\prime}\right)^{\prime}+\left(\mathrm{e}^{2 t}+\frac{1}{2} \mathrm{e}^{2 t+2}\right) x(t-3)=0 .
$$

It is not difficult to verify that all conditions of Theorem 2.2 are satisfied. Hence, equation (3.1) is oscillatory. However, the equation

$$
\left(\mathrm{e}^{2 t}\left(x(t)+\frac{1}{2} x(t-2)\right)^{\prime}\right)^{\prime}+\left(\mathrm{e}^{2 t}+\frac{1}{2} \mathrm{e}^{2 t+2}\right) x(t)=0
$$

has a nonoscillatory solution $x(t)=\mathrm{e}^{-t}$. Therefore, the delayed argument $\delta(t)=t-\delta$ plays an important role in oscillation of equation (1.1).

Remark 3.5 It would be of interest to find another method to study the equation

$$
\left(r(t)\left((x(t)+p(t) x(\tau(t)))^{\Delta}\right)^{\gamma}\right)^{\Delta}+q(t)|x(\delta(t))|^{\gamma-1} x(\delta(t))=0,
$$

where $p(t)<0$ or $p(t)>0$.

\section{Competing interests}

The authors declare that they have no competing interests.

\section{Authors' contributions}

All authors contributed equally to this paper and also read and approved the final manuscript.

\section{Author details}

${ }^{1}$ School of Information and Control Engineering, Weifang University, Weifang, Shandong 261061, P.R. China. ${ }^{2}$ Ramanujan Institute for Advanced Study in Mathematics, University of Madras, Chennai, 600 005, India.

\section{Acknowledgements}

The authors are grateful to reviewers for their comments and suggestions. This research is supported by Natural Science Foundation of Shandong Province (Z2007F08) and also by Weifang University Research Funds for Doctors (2012BS25). 


\section{References}

1. Hilger, S: Analysis on measure chains - a unified approach to continuous and discrete calculus. Results Math. 18 18-56 (1990)

2. Agarwal, RP, Bohner, M, O'Regan, D, Peterson, A: Dynamic equations on time scales: a survey. J. Comput. Appl. Math. $141,1-26(2002)$

3. Bohner, M, Peterson, A: Dynamic Equations on Time Scales: An Introduction with Applications. Birkhäuser, Boston (2001)

4. Bohner, M, Peterson, A: Advances in Dynamic Equations on Time Scales. Birkhäuser, Boston (2003)

5. Jiang, JC, Li, XP: Oscillation of second order nonlinear neutral differential equations. Appl. Math. Comput. 135, 531-540 (2003)

6. Saker, $\mathrm{SH}$ : New oscillation criteria for second-order nonlinear neutral delay difference equations. Appl. Math. Comput. $142,99-111(2003)$

7. Sun, YG, Saker, SH: Oscillation for second-order nonlinear neutral delay difference equations. Appl. Math. Comput. 163, 909-918 (2005)

8. Han, Z, Li, T, Sun, S, Zhang, C: Oscillation for second-order nonlinear delay dynamic equations on time scales. Adv. Differ. Equ. 2009, 1-13 (2009)

9. Agarwal, RP, O'Regan, D, Saker, SH: Oscillation criteria for second-order nonlinear neutral delay dynamic equations. J. Math. Anal. Appl. 300, 203-217 (2004)

10. Erbe, L, Hassan, TS, Peterson, A: Oscillation criteria for nonlinear functional neutral dynamic equations on time scales. J. Differ. Equ. Appl. 15, 1097-1116 (2009)

11. Şahiner, Y: Oscillation of second order neutral delay and mixed type dynamic equations on time scales. Adv. Differ. Equ. 2006, 1-9 (2006)

12. Saker, SH: Oscillation of second-order nonlinear neutral delay dynamic equations on time scales. J. Comput. Appl. Math. 187, 123-141 (2006)

13. Saker, SH, Agarwal, RP, O'Regan, D: Oscillation results for second-order nonlinear neutral delay dynamic equations on time scales. Appl. Anal. 86, 1-17 (2007)

14. Saker, $\mathrm{SH}, \mathrm{O}$ 'Regan, D: New oscillation criteria for second-order neutral functional dynamic equations via the generalized Riccati substitution. Commun. Nonlinear Sci. Numer. Simul. 16, 423-434 (2011)

15. Tripathy, AK: Some oscillation results for second order nonlinear dynamic equations of neutral type. Nonlinear Anal. 71, e1727-e1735 (2009)

16. Chen, DX: Oscillation of second-order Emden-Fowler neutral delay dynamic equations on time scales. Math. Comput. Model. 51, 1221-1229 (2010)

17. Zhang, SY, Wang, QR: Oscillation of second-order nonlinear neutral dynamic equations on time scales. Appl. Math. Comput. 216, 2837-2848 (2010)

18. Wu, HW, Zhuang, RK, Mathsen, RM: Oscillation criteria for second-order nonlinear neutral variable delay dynamic equations. Appl. Math. Comput. 178, 321-331 (2006)

19. Li, T, Agarwal, RP, Bohner, M: Some oscillation results for second-order neutral dynamic equations. Hacet. J. Math. Stat. 41, 715-721 (2012)

20. Li, T, Han, Z, Sun, S, Yang, D: Existence of nonoscillatory solutions to second-order neutral delay dynamic equations on time scales. Adv. Differ. Equ. 2009, 1-10 (2009)

21. Gao, C, Li, T, Tang, S, Thandapani, E: Oscillation theorems for second-order neutral functional dynamic equations on time scales. Electron. J. Differ. Equ. 2011, 1-9 (2011)

22. Baculíková, B, Džurina, J: Oscillation theorems for second-order nonlinear neutral differential equations. Comput. Math. Appl. 62, 4472-4478 (2011)

23. Zhang, C, Agarwal, RP, Bohner, M, Li, T: New oscillation results for second-order neutral delay dynamic equations. Adv. Differ. Equ. 2012, 1-14 (2012)

24. Candan, T: Oscillation criteria for second-order nonlinear neutral dynamic equations with distributed deviating arguments on time scales. Adv. Differ. Equ. 2013, 1-8 (2013)

25. Candan, T: Oscillation of second-order nonlinear neutral dynamic equations on time scales with distributed deviating arguments. Comput. Math. Appl. 62, 4118-4125 (2011)

26. Karpuz, B: Li type oscillation theorem for delay dynamic equations. Math. Methods Appl. Sci. 36, $993-1002$ (2013)

27. Şenel, MT: Kamenev-type oscillation criteria for the second-order nonlinear dynamic equations with damping on time scales. Abstr. Appl. Anal. 2012, 1-18 (2012)

28. Şenel, MT, Candan, T: Oscillation of second order nonlinear neutral differential equation. J. Comput. Anal. Appl. 14 1112-1117 (2012)

10.1186/1687-1847-2013-340

Cite this article as: Ji et al.: On oscillation of second-order neutral dynamic equations. Advances in Difference Equations 2013, 2013:340 